\title{
A descriptive study on multisystem inflammatory syndrome in children in a single center in West Michigan
}

\author{
Jonathan Shabab", Allysen Dubisky², Ambaris Singh', Megan Crippen ${ }^{1}$, Khalid Abulaban ${ }^{2,3}$ and Aileen Aldrich ${ }^{2,4^{*}}$ (D)
}

\begin{abstract}
Background: Multisystem Inflammatory Syndrome in Children (MIS-C) is a rare hyperinflammatory condition that occurs following SARS-CoV-2 infection. There is a paucity of research describing risk factors, optimal management, and outcomes of this life-threatening condition.
\end{abstract}

Methods: This is a case series of 26 patients diagnosed with MIS-C in a West Michigan pediatric tertiary care center from April 2020 to February 2021. We describe the clinical, imaging, and laboratory characteristics of these patients and detail their treatments and outcomes with comparisons between Pediatric Intensive Care Unit (PICU) and nonPICU patients. Categorical testing utilized Chi-square and Fisher's Exact tests. Comparison between groups used Ttests or Kruskal-Wallis.

Results: Fifteen patients (57\%) required intensive care. There was no statistically significant difference in demographics between PICU and non-PICU patients, however all Black patients required intensive care. Gastrointestinal symptoms were present in 22 patients (84\%). Seventeen patients (65\%) had Kawasaki-like features and $12(46 \%)$ developed coronary artery dilation. Patients requiring intensive care were less likely to have a reported history of COVID-19 disease or exposure $(p=0.0362)$. Statistically significant differences were also noted in peak ferritin $(p=0.0075)$, procalcitonin, and BNP in those who required intensive care.

Conclusions: Although overlap exists with other hyperinflammatory conditions, our study provides further evidence that MIS-C is a distinct, albeit heterogenous, disorder with various degrees of cardiac involvement. Anakinra, in conjunction with steroid use, appears to be effective and safe in the treatment of MIS-C. This report identifies procalcitonin, peak ferritin, and BNP as potentially useful biomarkers for severity of disease.

Keywords: MIS-C, Multisystem inflammatory syndrome in children, Pediatrics, COVID-19, SARS-CoV-2, Anakinra

\section{Background}

In late 2019, a cluster of viral pneumonia cases of novel coronavirus etiology was noted in Hubei Province, China, with the virus subsequently identified as SARS

\footnotetext{
* Correspondence: aileen.aldrich@helendevoschildrens.org

${ }^{2}$ Michigan State University College of Human Medicine, Grand Rapids, MI, USA

${ }^{4}$ Department of Pediatric Infectious Diseases, Spectrum Health Helen DeVos Children's Hospital, 35 Michigan St. NE, Suite 4150, Grand Rapids, Ml 49503, USA

Full list of author information is available at the end of the article
}

CoV-2 [1]. Early on, advanced age was recognized as a major risk factor for disease severity and death $[2,3]$. Conversely, children appeared relatively spared $[4,5]$. However, as SARS-CoV-2 spread across the world into a global pandemic [1], London researchers recognized a new entity [6], originally described as a severe Kawasakilike disease noted in children across Europe, including Italy and the United Kingdom [7, 8]. This later became known as Multisystem Inflammatory Syndrome in Children (MIS-C) in the United States [6]. Soon after, the

\section{BMC}

(c) The Author(s). 2021 Open Access This article is licensed under a Creative Commons Attribution 4.0 International License, which permits use, sharing, adaptation, distribution and reproduction in any medium or format, as long as you give appropriate credit to the original author(s) and the source, provide a link to the Creative Commons licence, and indicate if changes were made. The images or other third party material in this article are included in the article's Creative Commons licence, unless indicated otherwise in a credit line to the material. If material is not included in the article's Creative Commons licence and your intended use is not permitted by statutory regulation or exceeds the permitted use, you will need to obtain permission directly from the copyright holder. To view a copy of this licence, visit http://creativecommons.org/licenses/by/4.0/ The Creative Commons Public Domain Dedication waiver (http://creativecommons.org/publicdomain/zero/1.0/) applies to the data made available in this article, unless otherwise stated in a credit line to the data. 
Centers for Disease Control and Prevention (CDC) and the World Health Organization (WHO) developed case definitions for the diagnosis of MIS-C $[9,10]$.

In typical adult cases of COVID-19, patients present with fever, cough, and elevated inflammatory markers, often worsening in the second week of illness $[2,3]$. In severe cases, inflammatory markers continue to rise in association with acute respiratory distress syndrome and sepsis, with correspondent morbidity and mortality [3]. Children have been found to have a far lower risk of developing acute respiratory disease requiring hospitalization and intensive care, with the exception of children with specific underlying medical conditions such as obesity [11, 12]. Nevertheless, children are at greater risk of the post-infectious hyperinflammatory disease 2-8 weeks following asymptomatic or symptomatic infection with SARS-CoV-2 [13]. In accordance with the aforementioned case definitions, those with MIS-C have a persistent fever, at least two organ systems affected, and elevated inflammatory markers without alternate explanation $[9,10]$, although most patients have at least 4 organ systems involved [14]. Approximately 50\% of MIS-C patients have cardiac involvement severe enough to require vasoactive or vasopressor support, and in one study of 186 children, 4 died [14].

By February 28, 2021, there were nearly 625,000 confirmed cases of COVID-19 in Michigan [15]. Further, there were 99 reported cases of MIS-C by CDC criteria in the state $[9,16]$. At our institution, we developed a multidisciplinary protocol developed to assist in the diagnosis, evaluation, and treatment of MIS-C, modeled from American College of Rheumatology (ACR) guidelines [13]. Patients suspected of having MIS-C are evaluated by pediatric infectious disease and pediatric rheumatology in addition to the pediatric hospitalist and/or intensivist teams. Initial labs and work-up were obtained in accordance with our institutional algorithm (see details in Methods section below and Additional file 1: Appendix A). During hospitalization, MIS$\mathrm{C}$ patients are routinely monitored with daily inflammatory, cardiac, and coagulation labs. Additional subspecialty consults were provided as indicated. Our treatment consists of supportive care, anticoagulation with aspirin, low-molecular weight heparin, or heparin, and immunomodulatory agents including intravenous immunoglobulin (IVIG), steroids, and anakinra, as determined by the multidisciplinary team. Here, we report the clinical aspects, patterns, and outcomes observed at our institution between April 2020 and February 2021 while caring for children with MIS-C.

\section{Methods}

This study was a case series at Spectrum Health Helen DeVos Children's Hospital (HDVCH), a 241-bed children's tertiary care center in Grand Rapids, Michigan, USA. Subjects were pediatric patients admitted to HDVCH between April 1, 2020, and February 28, 2021, with a diagnosis of MIS-C as made by the consulting teams in concordance with $\mathrm{CDC}$ case definitions. The study received a HIPAA waiver from the Spectrum Health Institutional Review Board.

Once identified as a possible case of MIS-C by clinical criteria, patients were evaluated with complete blood count with manual differential, comprehensive metabolic panel (CMP), as well as inflammatory markers (erythrocyte sedimentation rate [ESR], C-reactive protein [CRP], D-dimer, fibrinogen, and ferritin). Urinalysis was obtained to evaluate for sterile pyuria and identify alternative causes for fever and inflammation. COVID-19 testing obtained included nasopharyngeal PCR and serum immunoglobulin G (IgG), which at first was a total antibody anti-nucleocapsid assay and later included anti-spike protein IgG. Brands and versions of the PCR and IgG tests used evolved over time based on test availability and performance. Any patients admitted to the hospital with suspicion for MIS-C underwent further laboratory evaluation, including coagulation studies (prothrombin time $[\mathrm{PT}]$, internationalized normal ratio [INR], and partial thromboplastin time [PTT]), procalcitonin, triglycerides, lactate dehydrogenase (LDH), creatinine kinase, and peripheral blood smear. If initial COVID-19 PCR testing was negative, a full respiratory pathogen panel (if not already performed), blood culture, and urine culture were performed to identify alternative etiologies. Patients admitted to the hospital with suspicion of MIS-C had daily labs, trending complete blood counts with manual differential, CMP, ESR, CRP, Ddimer, ferritin, and fibrinogen.

Initial cardiac evaluation for patients presenting with possible MIS-C included EKG and high sensitivity troponin. Following admission, patients were evaluated with brain natriuretic peptide (BNP) levels, and an echocardiogram was performed. BNP and troponin were repeated daily during admission. Echocardiograms were repeated based on clinical features, whether an alternate diagnosis was made, or as recommended by pediatric cardiology. Patients with cardiac abnormalities were followed by pediatric cardiology both during and after hospitalization with serial imaging.

Comparisons were performed on PICU versus nonPICU patients as a surrogate for disease severity. All statistical analyses were completed using SAS Enterprise Guide version 7.1 (SAS Institute Inc., Cary, NC, USA). Normality was assessed using a Shapiro Wilk test. Differences in categorical data were analyzed using Chisquare tests or Fisher's-Exact tests. For normally distributed and continuous data, means and standard deviation were described and T-tests were used to evaluate for 
significant differences. For non-normally distributed and continuous data, medians and interquartile values were established and Kruskal-Wallis tests were used to compare medians. For all analyses, $p$ values of $\leq 0.05$ were considered significant.

\section{Results}

There were 26 children diagnosed with MIS-C associated with COVID-19 at our institution between April 1, 2020, and February 28, 2021, which accounted for about one-third of the total cases in our state (Fig. 1). Of these, 15 (57\%) children required admission to the pediatric intensive care unit (PICU) designated as group 1. Those who did not require care in the PICU were designated as group 2. No statistically significant differences were noted in patient demographics which are delineated in table one. The clinical characteristics and cardiac findings of these 26 children are outlined in Table 1. Three patients had diagnosis of obesity prior to admission.

Every patient presented with fever, in accordance with initial diagnostic criteria. Gastrointestinal symptoms were common among these children, with 22/26 (84.6\%) experiencing abdominal pain, including 14/15 (93.3\%) of PICU cases. Kawasaki-like features (defined as having one or more of the major diagnostic clinical criteria of Kawasaki disease, namely bilateral conjunctivitis, unilateral lymphadenopathy of $1.5 \mathrm{~cm}$ or greater, rash, oral mucosa changes, or extremity changes) were seen in $17 /$ 26 children (65.4\%), 9 of which did not require PICU admission. Coronary dilation (Z-scores $\geq 2.0$ on echocardiogram) was seen in 12/26 children (46.2\%), 6 of whom required intensive care and 6 of whom did not. Dilation was seen in the left main coronary, circumflex, left coronary, left anterior descending and right coronary arteries. Other cardiac changes were seen in $15 / 26$ children (57.7\%), which included depressed left and/or right ventricle systolic function, mitral and tricuspid regurgitation, aortic root dilation, main pulmonary artery enlargement and pericardial effusion.

There were 17 children with a known history of COVID-19 infection or exposure; of these, 7 were in the PICU group and 10 in the non-PICU group, meaning patients in the PICU were less likely to have a COVID19 history $(p=0.0362)$. The COVID-19 anti-spike antibody was found in nearly all children (25/26, 96.2\%). Only one patient lacked anti-spike antibodies, and this patient was in the non-PICU group. Among the 16 children who had cytokine evaluations (10 in the PICU group, 6 in the non-PICU group), 100\% had elevation in IL-10. The PICU group included all children who needed any respiratory support, typically related to cardiorespiratory compromise $(12 / 26,46.2 \%)$. There were 2/15 (13.3\%) who required nasal cannula, $2 / 15$ (13.3\%) who required bilevel positive airway pressure, and $8 / 15$ (53.3\%) who required intubation amongst all the PICU cases. Multiple imaging studies were ordered on the patients in this study depending on their clinical

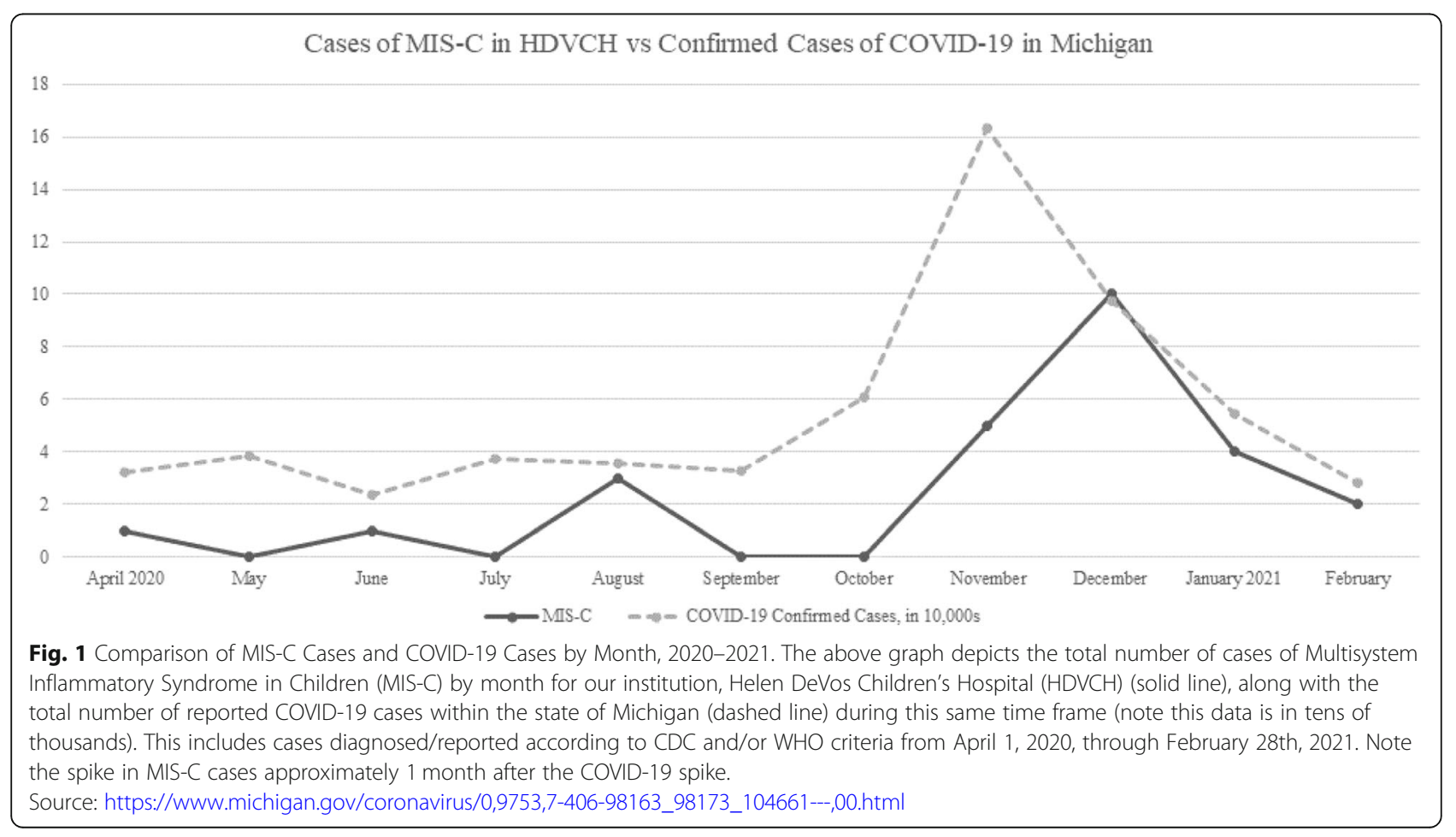


Table 1 Clinical characteristic and cardiac findings in patients with Multisystem Inflammatory Syndrome in Children in Michigan stratified by ICU admission, April 2020-February 2021

\begin{tabular}{|c|c|c|c|c|c|}
\hline \multicolumn{6}{|l|}{ Demographic Characteristics } \\
\hline \multirow[b]{2}{*}{ Total } & & \multirow{2}{*}{$\begin{array}{l}\text { Total }(\%) \\
26\end{array}$} & \multirow{2}{*}{$\begin{array}{l}\text { Group } 1 \text { (PICU) (\%) } \\
15\end{array}$} & \multirow{2}{*}{$\begin{array}{l}\text { Group } 2 \text { (non-PICU) (\%) } \\
11\end{array}$} & \multirow{2}{*}{$\begin{array}{l}p \\
\text { values }\end{array}$} \\
\hline & & & & & \\
\hline Age (months) & Mean (St Dev) & $105.15(57.3)$ & $99.13(53.4)$ & $113.36(64.0)$ & 0.5424 \\
\hline \multirow[t]{2}{*}{ Sex } & Male & $16(61.5)$ & $7(46.7)$ & $9(81.8)$ & 0.1092 \\
\hline & Female & $10(38.5)$ & $8(53.3)$ & $2(18.2)$ & \\
\hline \multirow[t]{5}{*}{ Race } & White & $14(53.5)$ & $8(53.3)$ & $6(54.6)$ & - \\
\hline & Black & $4(15.4)$ & $4(26.7)$ & $0(0)$ & - \\
\hline & Hispanic & $3(11.5)$ & $2(13.3)$ & $1(9.1)$ & - \\
\hline & Other & $3(11.5)$ & $0(0)$ & $3(27.3)$ & - \\
\hline & Unknown & $2(7.7)$ & $1(6.7)$ & $1(9.1)$ & - \\
\hline $\mathrm{BMI}$ & Median (IQR) & 18.095 (16.1-20.83) & $17.7(16.1-20.06)$ & 19.16 (15.44-22.98) & 0.5858 \\
\hline \multicolumn{6}{|l|}{ Clinical presentation } \\
\hline Fever & & $26(100)$ & $15(100)$ & $11(100)$ & 1.0 \\
\hline \multirow[t]{3}{*}{ Gl symptoms } & Vomiting & $19(73.1)$ & $12(80)$ & $7(63.6)$ & 0.4065 \\
\hline & Diarrhea & $14(53.9)$ & $7(46.7)$ & $7(63.6)$ & 0.3912 \\
\hline & Abdominal pain & $22(84.6)$ & $14(93.3)$ & $8(72.7)$ & 0.2789 \\
\hline Skin rash & & $15(57.7)$ & $8(53.3)$ & $7(63.6)$ & 0.7007 \\
\hline Other Kawasaki-like features ${ }^{a}$ & & $17(65.4)$ & $8(53.3)$ & $9(81.8)$ & 0.1315 \\
\hline \multirow[t]{2}{*}{ Cardiac involvement } & Coronary dilation & $12(46.2)$ & $6(40)$ & $6(54.6)$ & 0.4623 \\
\hline & Other cardiac changes & $15(57.7)$ & $11(73.3)$ & $4(36.4)$ & 0.1089 \\
\hline History of COVID-19 infection/exposure & & $17(65.4)$ & $7(46.7)$ & $10(90.9)$ & $0.0362^{*}$ \\
\hline \multirow[t]{9}{*}{ Cytokine elevation ${ }^{\mathrm{b}}$} & IL-2 Soluble & $8(50)$ & $4(40)$ & $4(66.7)$ & 0.6084 \\
\hline & IL-2 Receptor & $12(75)$ & $9(90)$ & $3(50)$ & 0.1181 \\
\hline & IL-5 & $2(12.5)$ & $2(20)$ & $0(0)$ & 0.5 \\
\hline & $\mathrm{IL}-6$ & $7(43.8)$ & $6(60)$ & $1(16.7)$ & 0.1451 \\
\hline & $\mathrm{IL}-10$ & $16(100)$ & $10(100)$ & $6(100)$ & \\
\hline & $\mathrm{IL}-13$ & $6(37.5)$ & $5(50)$ & $1(16.7)$ & 0.3069 \\
\hline & $\mathrm{IL}-17$ & $5(31.3)$ & $4(40)$ & $1(16.7)$ & 0.5879 \\
\hline & TNF-alpha & $3(18.8)$ & $2(20)$ & $1(16.7)$ & 1.0 \\
\hline & TNF-gamma & $6(37.5)$ & $4(40)$ & $2(33.3)$ & 1.0 \\
\hline Positive COVID-19 PCR on admission & & $11(42.3)$ & $6(40)$ & $5(45.5)$ & 1.0 \\
\hline \multirow[t]{2}{*}{ COVID-19 antibody } & Anti-Spike & $25(96.2)$ & $15(100)$ & $10(90.9)$ & 0.4231 \\
\hline & Anti-Nucleocapsid & $18(85.7)$ & $10(83.3)$ & $8(88.9)$ & 1.0 \\
\hline \multirow[t]{3}{*}{ Respiratory support } & Nasal cannula & $2(7.7)$ & $2(13.3)$ & $0(0)$ & - \\
\hline & BiPap & $2(7.7)$ & $2(13.3)$ & $0(0)$ & - \\
\hline & Intubated & $8(30.8)$ & $8(53.3)$ & $0(0)$ & - \\
\hline
\end{tabular}

BMI Body mass index, IQR interquartile range, GI Gastrointestinal, COVID-19 Coronavirus disease 2019, PCR polymerase chain reaction, IL Interleukin, TNF Tumor necrosis factor, BiPap Bilevel positive airway pressure

a cervical adenopathy, oral mucosa changes, peripheral extremity changes, and/or bilateral non-purulent conjunctivitis

$\mathrm{b}$ Incorporated into practice during study period, not universally obtained on all included patients

*significant $p$ value $(\leq 0.05)$

symptoms; details and statistical analysis were beyond the scope of this review.

All 26 patients underwent EKGs, 20 (76\%) of which were abnormal. The abnormal EKG findings included sinus tachycardia, sinus bradycardia, nonspecific ST segment and $\mathrm{T}$ wave changes, low voltage QRS complexes, poor $\mathrm{R}$ wave progression, prolonged $\mathrm{QT}$ interval, borderline prolonged PR interval, right axis deviation, left 
axis deviation, premature ventricular contractions, and possible right ventricular hypertrophy.

Laboratory data at presentation are outlined in Table 2. All patients had elevated inflammatory markers at presentation, which were necessary criteria for diagnosis of MIS-C. Other general lab findings included anemia, lymphopenia, elevated creatinine, mild elevation in AST and/or ALT, and elevated troponin and BNP levels. The PICU group had significantly higher procalcitonin levels with a median of $11.1 \mathrm{ng} / \mathrm{mL}$ while the non-PICU group had a median of $2.79 \mathrm{ng} / \mathrm{mL}(p=0.0298)$. The BNP level was significantly higher in the PICU group with a median of $7019 \mathrm{pg} / \mathrm{mL}$ compared to the non-PICU group with a median of $1179 \mathrm{pg} / \mathrm{mL}(p=0.0218)$. The ferritin level at presentation, although higher in the PICU group, was not statistically significantly higher than in the non-
PICU group; however, the peak ferritin level in the PICU group was significantly higher than the non-PICU group (median 1008 vs $270, p=0.0075$ ). The mean hemoglobin level in the PICU group was significantly lower at presentation than the non-PICU group $(10.31 \mathrm{~g} / \mathrm{dL}$ vs 12.05 $\mathrm{g} / \mathrm{dL}, p=0.0196)$. The median AST for the PICU group was higher than the non-PICU group $(39 \mathrm{U} / \mathrm{L}$ vs $22 \mathrm{U} / \mathrm{L}$, $p=0.0331$ ). The non-PICU group had higher LDH and CK medians than the PICU group, though not statistically significant.

The management of the 26 patients with MIS-C is outlined in Table 3. There were 25 children who received aspirin and 25 children who received IVIG ( $2 \mathrm{~g} /$ $\mathrm{kg}$ ), with only one child in the non-PICU group not receiving either due to a very mild clinical case. Severe presentations or those not clinically responsive to IVIG

Table 2 Laboratory data at presentation for patients with Multisystem Inflammatory Syndrome in Children in Michigan, April 2020 February 2021

\begin{tabular}{|c|c|c|c|c|c|}
\hline & Total & $\begin{array}{l}\text { Group } 1 \\
\text { (PICU) }\end{array}$ & $\begin{array}{l}\text { Group } 2 \\
\text { (non-PICU) }\end{array}$ & $\begin{array}{l}\text { Reference } \\
\text { value }\end{array}$ & $\begin{array}{l}p \\
\text { values }\end{array}$ \\
\hline Total & 26 & 15 & 11 & & \\
\hline WBC (K/CUMM) & 10.385 (8.76-13.03) & $10.70(8.76-14.46)$ & $9.51(7.65-11.6)$ & $5.00-15.00$ & 0.4062 \\
\hline $\mathrm{Hgb}(\mathrm{g} / \mathrm{dL})^{\mathrm{a}}$ & $11.04(1.93)$ & $10.31(1.84)$ & $12.05(1.62)$ & $11.5-14.5$ & $0.0196^{*}$ \\
\hline Platelets (K/CUMM) & $179.5(137-226)$ & 147 (124-209) & $183(158-250)$ & $140-400$ & 0.2128 \\
\hline ALC (K/CUMM) & $880.5(402-1490)$ & 876 (299-1490) & 1073 (580-1866) & $800-7900$ & 0.3781 \\
\hline Sodium $(\mathrm{mMol} / \mathrm{L})^{\mathrm{a}}$ & $133.73(5.44)$ & $134.27(6.53)$ & $133(3.66)$ & $134-146$ & 0.5685 \\
\hline Bicarbonate $(\mathrm{mMol} / \mathrm{L})$ & $22(19-24)$ & $22(19-24)$ & $24(19-24)$ & $21-29$ & 0.4951 \\
\hline Creatinine (mg/dL) & $0.475(0.33-0.70)$ & $0.48(0.32-0.70)$ & $0.41(0.33-0.76)$ & $<0.6$ & 0.8762 \\
\hline Albumin $(\mathrm{g} / \mathrm{dL})^{\mathrm{a}}$ & $2.76(0.64)$ & $2.62(0.69)$ & $2.95(0.54)$ & $3.5-5.0$ & 0.2070 \\
\hline $\mathrm{ALT}(\mathrm{U} / \mathrm{L})$ & $26(16-39)$ & $27(16-51)$ & $25(13-37)$ & $<40$ & 0.5853 \\
\hline AST (U/L) & $32(21-44)$ & $39(25-70)$ & $22(17-39)$ & $<40$ & $0.0331^{*}$ \\
\hline $\mathrm{LDH}(\mathrm{U} / \mathrm{L})$ & $280.5(222.5-341)$ & $267(217-334)$ & $327(228-348)$ & $<271$ & 0.4516 \\
\hline Triglycerides (mg/dL) & $131(103-185)$ & $130(102-185)$ & $132(124-153)$ & $<150$ & 0.8053 \\
\hline $\operatorname{ESR}(\mathrm{mm} / \mathrm{h})^{\mathrm{a}}$ & $48.12(30.4)$ & $44.33(29.59)$ & $53.27(32.16)$ & $<13$ & 0.4701 \\
\hline CRP (mg/L) & $110.3(82.6-164.9)$ & $145(95.1-177.9)$ & $90.9(76-110.3)$ & $<5$ & 0.0616 \\
\hline Procalcitonin (ng/ml) & $6.74(2.78-16.84)$ & $11.1(5.7-32.8)$ & $2.79(1.52-4.98)$ & $<0.25$ & $0.0298^{*}$ \\
\hline Ferritin (ng/mL) & $374(178-809)$ & $520(178-1590)$ & $200(178-604.2)$ & $<336$ & 0.1611 \\
\hline Peak Ferritin (ng/mL) & $690.5(274-1214)$ & 1008 (513-1733) & $270(200-829)$ & $7-142$ & $0.0075^{*}$ \\
\hline D-dimer (mg/l FEU) & $2190(1030-3800)$ & $2355(1540-6850)$ & $2190(690-2810)$ & $<0.5$ & 0.2983 \\
\hline $\mathrm{BNP}(\mathrm{pg} / \mathrm{mL})$ & $2147(552-11,023)$ & $7019(1631-20,170)$ & $1179(245-2147)$ & $<101$ & $0.0218^{*}$ \\
\hline Troponin (ng/L) & $18(5.99-40)$ & $18(5.99-40)$ & $13(5.99-45)$ & $<17$ & 0.6739 \\
\hline $\mathrm{CK}(\mathrm{U} / \mathrm{L})$ & $73.5(43-215)$ & $59(43-268)$ & $88(24-162)$ & $<233$ & 0.5518 \\
\hline Fibrinogen $(\mathrm{mg} / \mathrm{dL})^{\mathrm{a}}$ & $505.27(127.51)$ & $496.27(129.8)$ & $517.55(129.51)$ & $190-400$ & 0.6830 \\
\hline $\mathrm{PTT}(\mathrm{s})$ & $26(23.5-28)$ & $26(24-29)$ & $26.5(23-27)$ & $<33.1$ & 0.9765 \\
\hline INR & $1.1(1.1-1.2)$ & $1.2(1.1-1.6)$ & $1.1(1-1.2)$ & $<1.1$ & 0.1121 \\
\hline
\end{tabular}

$I Q R$ Interquartile range, WBC White blood cells, Hgb Hemoglobin, ALC Absolute lymphocyte count, ALT Alanine aminotransferase, AST Aspartate aminotransferase, $L D H$ Lactate dehydrogenase, ESR Erythrocyte sedimentation rate, CRP C-reactive protein, BNP Brain natriuretic peptide, $C K$ Creatine kinase, $P T T$ Partial thromboplastin time, INR International normalized ratio

${ }^{2}$ These were normally distributed data, therefore mean and standard deviations are reported. The remainder of the data was not normally distributed and so medians and interquartile ranges are reported

*significant $p$ value $(\leq 0.05)$ 
Table 3 Management of 26 patients with Multisystem Inflammatory Syndrome in Children in Michigan, April 2020 - February 2021

\begin{tabular}{|c|c|c|c|c|}
\hline \multicolumn{5}{|l|}{ Treatment } \\
\hline \multirow[b]{2}{*}{ Total } & \multirow{2}{*}{$\begin{array}{l}\text { Total (\%) } \\
26\end{array}$} & \multirow{2}{*}{$\begin{array}{l}\text { Group } 1 \text { (PICU) (\%) } \\
15\end{array}$} & \multirow{2}{*}{$\begin{array}{l}\text { Group } 2 \text { (non-PICU) (\%) } \\
11\end{array}$} & \multirow{2}{*}{$\begin{array}{l}p \\
\text { values }\end{array}$} \\
\hline & & & & \\
\hline Inotropes $^{a}$ & $12(46.2)$ & $12(80)$ & $0(0)$ & $0.0001^{*}$ \\
\hline Aspirin & $25(96.2)$ & $15(100)$ & $10(90.9)$ & 0.4231 \\
\hline Low-molecular weight heparin & $12(46.2)$ & $9(60)$ & $3(27.3)$ & 0.0982 \\
\hline Heparin & $5(19.2)$ & $5(33.3)$ & $0(0)$ & 0.0527 \\
\hline IVIG & $25(96.2)$ & $15(100)$ & $10(90.9)$ & 0.4231 \\
\hline Steroids & $22(84.6)$ & $13(86.7)$ & $9(81.8)$ & 1.0 \\
\hline Steroid pulse & $7(26.9)$ & $6(40)$ & $1(9.1)$ & 0.1783 \\
\hline Anakinra & $13(50)$ & $10(66.7)$ & $3(27.3)$ & $0.0472^{*}$ \\
\hline Infliximab & $1(3.9)$ & $1(6.7)$ & $0(0)$ & 1.0 \\
\hline
\end{tabular}

IVIG Intravenous immune globulin

${ }^{a}$ This included epinephrine, norepinephrine, vasopressin, and/or milrinone

*significant $p$ value $(\leq 0.05)$

were additionally treated with IV methylprednisolone, either low dose $(2 \mathrm{mg} / \mathrm{kg} /$ day as a single dose or divided twice a day) or pulse dosing $(15-30 \mathrm{mg} / \mathrm{kg}$ with maximum of 1000 once daily for $3-5$ days). Corticosteroids were given to 22 children, 13 of whom were in the PICU. Seven children received pulse corticosteroids, only 1 of whom was outside the PICU. Anakinra was given more frequently in the PICU group compared to the non-PICU group (66.7 vs $27.3 \%, p=0.0472$ ).

Of those admitted to intensive care, 12 out 15 (80\%) required inotropes (epinephrine, norepinephrine, vasopressin, and/or milrinone). Anticoagulation with low molecular weight heparin or heparin was given to 9 of 12 children (60\%) in the PICU group and 5 children in the non-PICU group; all 5 children who received heparin were in the PICU group. Infliximab was given to one child $(3.9 \%)$ that was in the PICU group and was diagnosed with MIS-C retrospectively based on clinical characteristics but at the time (April 2020) was treated as Kawasaki Shock Syndrome. One patient received antiviral treatment with remdesivir due to concern for concurrent acute COVID-19 infection and marked severity of illness who subsequently expired.

Concurrent acute bacterial infections occurred in $2 \mathrm{pa}-$ tients (8\%), including a urinary tract infection in one and another with a central line-associated bloodstream infection complicated by peritonitis. Other complications included extracorporeal membrane oxygenation (ECMO) use in 3 patients (12\%), ischemic cerebrovascular accident or intracranial hemorrhage in 2 patients (8\%), bilateral upper and lower extremity amputations in 1 patient (4\%), persistent first-degree atrioventricular (AV) block in 1 patient (4\%), requirement of enteral tube feeds at discharge in 1 patient (4\%), disseminated intravascular coagulation in 1 patient (4\%), and death in 1 patient (4\%). Median length of stay was 6 days (range 2-43 days) overall, with longer admissions for those in the PICU group compared to the non-PICU group ( 8 days vs 5 days, $p=0.002$ ).

All discharged patients had pediatric specialty followup with cardiology, infectious diseases, and rheumatology. Patients treated with corticosteroids completed a 26 week oral taper after discharge as per ACR guidelines; weans were performed at the discretion of the treating rheumatologist in light of relevant clinical or laboratory abnormalities. The duration of anakinra varied widely from only a few days during their inpatient stay to several weeks past discharge. Increased institutional experience in association with reports from other groups over time allowed for reduction of total duration of anakinra therapy. One patient required readmission for recrudescent fevers and increase in inflammatory markers after completing a 6-week oral corticosteroid taper. It is worth noting that the initial presentation was consistent with MIS-C. Anakinra therapy was continued for 3 months. Trial off resulted in clinical and laboratory recurrence of inflammatory features. Subsequently she transitioned to canakinumab (IL-1 antagonist) and is currently clinically well. This patient did undergo further investigative work up looking for potential other autoinflammatory disease which was non-diagnostic and has whole genome sequencing studies pending.

\section{Discussion}

\section{Overall findings}

From a demographic standpoint, ethnicity and race have been reported to impact the severity of disease in those affected by acute respiratory COVID-19 due to a variety of proposed mechanisms $[17,18]$. The same effect of race/ethnicity on disease severity has not yet been demonstrated in MIS-C. In this report, we did not find a statistically significant difference in demographics and 
outcomes. However, it should be noted that all Black patients included in this study were admitted to the PICU, and that this group included the only mortality as well as the patient with the most significant morbidity (amputations). One unusual finding in comparing the PICU vs non-PICU group features is the lower incidence of a reported prior COVID-19 history in patients admitted to the PICU. Whether this relates to more rapid immune dysregulation $[19,20]$, delayed presentation to care, or concomitant acute manifestations of COVID-19 is unclear and further studies with larger cohorts are needed.

\section{Lab differences}

Statistically significant differences were found between the PICU and non-PICU groups regarding laboratory values of hemoglobin, BNP, AST, peak ferritin, and procalcitonin. These laboratory derangements correlated with disease severity in our cohort.

Mean hemoglobin was found to be lower in the PICU group; however, the interpretation of this finding is uncertain, especially given that normal ranges for hemoglobin vary by age. Anemia has been frequently described in critical care settings as well in inflammatory states, and the etiology of anemia in our cohort may be multifactorial: iatrogenic (more frequent blood draws, dilution from fluid resuscitation), anemia of acute inflammation, and possibly preexisting anemia of iron deficiency [21]. Our small cohort and retrospective study design do not allow us to delineate between these potential causes. On average, BNP was greater in the PICU group than the non-PICU group. Elevated BNP correlates with greater cardiac dysfunction, and indeed may correlate with disease severity.

In the PICU group, we observed greater elevation in AST, peak ferritin, and procalcitonin. The elevation in AST may be secondary to the greater organ dysfunction and shock present in the PICU group [22]. Elevations in ferritin are commonly associated with inflammatory and immune dysregulation states. This, in combination with the increased AST, is reminiscent of hemophagocytic lymphohistiocytosis and macrophage activation syndrome (HLH/MAS). These immune dysregulation disorders have previously been reported secondary to viral illness [22] and so it may follow that MIS-C may have a similar pathophysiological basis with SARS-CoV-2 as a trigger. Procalcitonin was also substantially elevated in the ICU patients; this may be a biomarker of multiorgan dysfunction, as excretion of procalcitonin is mediated by the kidneys. However, procalcitonin was elevated in patients who did not have documented renal dysfunction, and may therefore be a biomarker for disease severity in MIS-C.

\section{Treatment approaches}

Most patients that underwent treatment for MIS-C received IVIG as per our institutional guidelines. Corticosteroids were used as adjuvant therapy in the majority of patients unless presentation was very mild. Pulse dose corticosteroids were used in patients with critical illness requiring inotropic medication and positive pressure ventilation, or extensive changes on echo including extensive coronary artery dilation or clinically significant effusion. Those with severe presentation, continued decline, or poor response to other therapies were additionally treated with anakinra. Statistically significant differences were found between the PICU and nonPICU groups with usage of inotropes and anakinra. Our institution restricts inotropes to the critical care or emergency department setting, and the majority of the patients cared for in the PICU required inotropic support. We followed the suggestion of early ACR guidelines for anakinra implementation in MIS-C. Our greater reliance on anakinra is likely attributable to the pediatric rheumatology department's familiarity with using anakinra in managing diseases like systemic juvenile idiopathic arthritis (JIA) [23]. Anakinra is a recombinant interleukin 1 (IL-1) receptor antagonist that is more commonly used to treat autoinflammatory diseases including systemic JIA, cryopyrin-associated periodic fever syndromes and even HLH/MAS [24, 25]. A common pathogenesis of those diseases is an exaggerated innate immune response with the hallmark of an amplified IL-1 cytokine signaling $[24,25]$.

The principal distinguishing feature of patients who required intensive care was the degree of shock. Our cohort's patients cared for in the PICU had corresponding elevation in BNP, as well as a hyperinflammatory state demonstrated by elevations in AST, peak ferritin and procalcitonin, which is similar to prior literature [12].

\section{Outcomes}

The majority of patients diagnosed with MIS-C at our institution in the study period were discharged from the hospital in good condition without apparent persistent sequalae. However, several of the patients included in the study group had lingering cardiac disease including coronary dilation, cardiac dysfunction, or both at discharge. We had only one patient with persistent 1st degree AV block after discharge that required prolonged aspirin therapy. The long-term cardiac sequelae of patients diagnosed along the spectrum of severity of MIS$\mathrm{C}$ are yet to be determined [26-28], and chronic followup with Pediatric Cardiology is planned for our cohort.

However, some patients did experience substantial morbidity during their hospitalization for MIS-C. In particular, one patient had severe shock and cardiovascular collapse necessitating ECMO. During this time, he 
developed ischemic injury secondary to edema and disseminated intravascular coagulation leading to dry gangrene of all distal extremities necessitating amputation. He additionally developed profound multiple organ dysfunction and required CRRT, with subsequent hemodialysis. Prior medical history was notable for autism, obesity, and mild intermittent asthma. Unfortunately, our cohort also included one patient death. This patient experienced severe shock with cardiogenic, septic, and distributive features. After a brief period of clinical stability, the patient experienced rapid deterioration and was found to have superimposed gram-negative septicemia. This subsequently led to ECMO due to catecholamine resistant shock. This was complicated by profound intracranial hemorrhage and the patient's family elected to withdraw technological support. The etiology of the patient's superimposed bloodstream infection is uncertain, although multiple mechanisms were proposed. It is possible that the patient's degree of shock led to microangiopathic ischemic changes to the intestinal mucosa that allowed for dissemination of enteric bacteria into the patient's bloodstream. Further, the patient was substantially immunosuppressed with both pulse and high dose corticosteroids, and anakinra, making her more susceptible to opportunistic infection. Both selected severe cases did occur in Black children.

Notably, as above, our data does suggest that concomitant infections can and do occur in MIS-C. This occurred in two of the patients in our cohort. Whether the hyperinflammatory state predisposes individuals to be at risk of developing other infections or this is subsequent to the effects of immunomodulating medications is unclear. Of note, anakinra has not been associated with secondary infections in prior research on chronic rheumatological conditions [29-31]. Observational studies have demonstrated a dose dependent effect of infection associated with corticosteroid use; however, this has not been replicated with randomized controlled trials [32].

\section{Addition to the literature}

The patients presented in this cohort add to the growing understanding of MIS-C as a potentially life-threatening disease process with a spectrum of cardiac, hematologic and immunologic manifestations. Characteristics of the disease mimic that of other diseases known or believed to be the result of immune dysregulation.

All the patients in our study did have at least some features that overlap with Kawasaki disease, HLH, MAS and other acute immune dysregulation disorders as has been reported in other studies [33]. However, the diagnostic criteria for these conditions were not met in all patients. Therefore, our study adds to the increasing body of evidence that, although apparently related to these other conditions, MIS-C is a distinct disorder with possible overlapping genetic tendencies [34, 35].

Relative to other institutions, our approach to MIS-C generally relied on corticosteroids and anakinra and a collaborative multidisciplinary approach was utilized for diagnosis and management of those patients. For PICU patients, we aimed for complete multidisciplinary rounds daily, when possible, which encouraged real-time discussion of individual cases amongst all involved specialists.

In our study group, no adverse outcomes were noted that were directly attributable to Anakinra use. Given the manner in which we performed our study we cannot directly determine benefit. However, there is a biological plausibility to support its use and accordingly, we believe that randomized controlled trials should be performed.

Some of the laboratory findings in MIS-C that our case series found are reminiscent of an HLH/MAS-like cytokine storm (elevated ferritin, pancytopenia, elevated inflammatory markers). However, the degree of inflammation was not as marked as is typically noted in HLH/ MAS, leading credence to MIS-C as a separate disease process with potentially overlapping pathogenesis. Outcomes in our patients were overall favorable while using anakinra which suggests it has a role in management of severe MIS-C when refractory to IVIG and corticosteroids.

\section{Study limitations}

MIS-C is a rare [36] but important syndrome to identify and treat, in light of its widely varying symptomatology and disease severity. Our institution only diagnosed those severe enough to require hospital admission. Our awareness and understanding of the disease evolved as our institutional knowledge increased. This extended to include the diagnostic criteria that were used in conjunction with $\mathrm{CDC}$ and $\mathrm{WHO}$ guidelines to identify those patients with MIS-C. Given this change in diagnostic criteria it is possible that patients would have been included or excluded differently at different points within the study period.

\section{Future study}

Identification of precise biomarkers that predict severe disease, to quickly identify those patients who may need escalation of care or additional therapeutics, is needed. Although our sample size is low, we suggest that significantly elevated ferritin and procalcitonin may predict a worse disease course.

More research needs to be done to improve our understanding of the patient population that is at the highest risk for developing MIS-C, which remains a rare complication in children after acute COVID-19 infection. 


\section{Conclusion}

Although overlaps exist with other hyperinflammatory conditions, our study provides further evidence that MIS-C is a distinct, albeit heterogenous, disorder with possible overlapping features with Kawasaki, MAS, and HLH. Anakinra, in conjunction with corticosteroids, IVIG, and supportive care, is demonstrated to be worthy of evaluation as an adjuvant agent in the treatment of MIS-C. Further, this report identifies procalcitonin, peak ferritin, and BNP as potentially useful markers for severity of disease.

\begin{abstract}
Abbreviations
ACR: American College of Rheumatology; AST: Aspartate Aminotransferase; BNP: Brain Natriuretic Peptide; CDC: Centers for Disease Control and Prevention; CMP: Comprehensive Metabolic Panel; COVID-19: Coronavirus Disease 2019; CRP: C-Reactive Protein; ECMO: Extracorporeal membrane oxygenation; EKG: Electrocardiogram; ESR: Erythrocyte Sedimentation Rate; HDVCH: Spectrum Health Helen DeVos Children's Hospital; HIPAA: Healthcare Information Portability and Accountability Act; HLH: Hemophagocytic Lymphohistiocytosis; IL: Interleukin; IVIG: Intravenous Immunoglobin; JIA: Juvenile idiopathic arthritis; MAS: Macrophage Activation Syndrome; MISC: Multisystem Inflammatory Syndrome in Children; PCR: Polymerase chain reaction; PICU: Pediatric Intensive Care Unit; SARS-CoV-2: Severe Acute Respiratory Syndrome Coronavirus 2; WHO: World Health Organization
\end{abstract}

\section{Supplementary Information}

The online version contains supplementary material available at https://doi. org/10.1186/s12969-021-00658-3.

Additional file 1: Appendix A. Institutional MIS-C Algorithm.

\section{Acknowledgements}

Special thanks to our colleague Dr. Rosemary Olivero for editing of this manuscript.

\section{Authors' contributions}

AA created the project and worked with KA and JS on study design. AA, MC, $A S$, and $A D$ all contributed to writing methods and obtaining IRB approval. Data was collected by JS, AD, MC, AS and updated by AA as needed. AA oversaw data analysis. Each author contributed to sections of the manuscript, but majority of discussion was written by JS, AA, and KA. AD created the tables and JS made the figure. All authors read and approved the final manuscript.

\section{Funding}

There was no funding for this project.

\section{Availability of data and materials}

The datasets generated and/or analyzed during the current study are not publicly available but are available from the corresponding author on reasonable request.

\section{Declarations}

Ethics approval and consent to participate

This project received approval from the Spectrum Health Institutional Review Board and a waiver of consent was granted.

\section{Consent for publication}

Not applicable.

\section{Competing interests}

The authors declare that they have no competing interests.

\section{Author details}

${ }^{1}$ Spectrum Health Helen DeVos Children's Hospital/Michigan State University Pediatric Residency Program, Grand Rapids, MI, USA. ${ }^{2}$ Michigan State University College of Human Medicine, Grand Rapids, MI, USA. ${ }^{3}$ Department of Pediatric Rheumatology, Spectrum Health Helen DeVos Children's Hospital, Grand Rapids, MI, USA. ${ }^{4}$ Department of Pediatric Infectious Diseases, Spectrum Health Helen DeVos Children's Hospital, 35 Michigan St. NE, Suite 4150, Grand Rapids, MI 49503, USA.

Received: 27 September 2021 Accepted: 28 November 2021

Published online: 16 December 2021

\section{References}

1. Huang C, Wang Y, Li X, Ren L, Zhao J, Hu Y, et al. Clinical features of patients infected with 2019 novel coronavirus in Wuhan, China. Lancet. 2020;395(10223):497-506.

2. Guan W-J, Ni Z-Y, Hu Y, Liang W-H, Ou C-Q, He J-X, et al. Clinical characteristics of coronavirus disease 2019 in China. N Engl J Med. 2020; 382(18):1708-20.

3. Zhou F, Yu T, Du R, Fan G, Liu Y, Liu Z, et al. Clinical course and risk factors for mortality of adult inpatients with COVID-19 in Wuhan, China: a retrospective cohort study. Lancet. 2020;395(10229):1054-62.

4. Zimmermann P, Curtis N. COVID-19 in children, pregnancy and neonates: a review of epidemiologic and clinical features. Pediatr Infect Dis J. 2020;39(6): 469-77.

5. Davies NG, Klepac P, Liu Y, Prem K, Jit M, CMMID COVID-19 working group, et al. Age-dependent effects in the transmission and control of COVID-19 epidemics. Nat Med. 2020;26(8):1205-11.

6. Riphagen S, Gomez X, Gonzalez-Martinez C, Wilkinson N, Theocharis P. Hyperinflammatory shock in children during COVID-19 pandemic. Lancet. 2020;395(10237):1607-8

7. Whittaker E, Bamford A, Kenny J, Kaforou M, Jones CE, Shah P, et al. Clinical characteristics of 58 children with a pediatric inflammatory multisystem syndrome temporally associated with SARS-CoV-2. JAMA. 2020;324(3):25969.

8. Viner RM, Whittaker E. Kawasaki-like disease: emerging complication during the COVID-19 pandemic. Lancet. 2020;395(10239):1741-3.

9. HAN Archive - 00432 | Health Alert Network (HAN) [Internet]. [cited 2021 Jul 2]. Available from: https://emergency.cdc.gov/han/2020/han00432.asp

10. Multisystem inflammatory syndrome in children and adolescents temporally related to COVID-19 [Internet]. [cited 2021 Jul 2]. Available from: https:// www.who.int/news-room/commentaries/detail/multisystem-inflammatorysyndrome-in-children-and-adolescents-with-covid-19

11. Siebach MK, Piedimonte G, Ley SH. COVID-19 in childhood: transmission, clinical presentation, complications and risk factors. Pediatr Pulmonol. 2021; 56(6):1342-56.

12. Abrams JY, Oster ME, Godfred-Cato SE, Bryant B, Datta SD, Campbell AP, et al. Factors linked to severe outcomes in multisystem inflammatory syndrome in children (MIS-C) in the USA: a retrospective surveillance study. Lancet Child Adolesc Health. 2021;5(5):323-31.

13. Henderson LA, Canna SW, Friedman KG, Gorelik M, Lapidus SK, Bassiri H, et al. American College of Rheumatology Clinical Guidance for multisystem inflammatory syndrome in children associated with SARS-CoV-2 and Hyperinflammation in pediatric COVID-19: version 1. Arthritis Rheumatol. 2020;72(11):1791-805.

14. Feldstein LR, Rose EB, Horwitz SM, Collins JP, Newhams MM, Son MBF, et al. Multisystem inflammatory syndrome in U.S. children and adolescents. N Engl J Med. 2020;383(4):334-46.

15. Coronavirus - Coronavirus [Internet]. [cited 2021 Jul 2]. Available from: https://www.michigan.gov/Coronavirus

16. Coronavirus - MIS-C Data and Reporting [Internet]. [cited 2021 Jul 2]. Available from: https://www.michigan.gov/coronavirus/0,9753,7-406-98163_ 98173_104661\%2D\%2D-,00.html

17. Gao Y-D, Ding M, Dong X, Zhang J-J, Kursat Azkur A, Azkur D, et al. Risk factors for severe and critically ill COVID-19 patients: a review. Allergy. 2021; 76(2):428-55

18. Price-Haywood EG, Burton J, Fort D, Seoane L. Hospitalization and mortality among black patients and white patients with Covid-19. N Engl J Med. 2020;382(26):2534-43.

19. Ramaswamy A, Brodsky NN, Sumida TS, Comi M, Asashima H, Hoehn KB, et al. Immune dysregulation and autoreactivity correlate with disease 
severity in SARS-CoV-2-associated multisystem inflammatory syndrome in children. Immunity. 2021;54(5):1083-1095.e7.

20. Matic KM. SARS-CoV-2 and multisystem inflammatory syndrome in children (MIS-C). Curr Probl Pediatr Adolesc Health Care. 2021:51(4):101000.

21. Prakash D. Anemia in the ICU: anemia of chronic disease versus anemia of acute illness. Crit Care Clin. 2012;28(3):333-43.

22. Canna SW, Marsh RA. Pediatric hemophagocytic lymphohistiocytosis. Blood. 2020;135(16):1332-43.

23. McMurray JC, May JW, Cunningham MW, Jones OY. Multisystem inflammatory syndrome in children (MIS-C), a post-viral myocarditis and systemic Vasculitis-a critical review of its pathogenesis and treatment. Front Pediatr. 2020;8:626182.

24. Sönmez HE, Demir S, Bilginer Y, Özen S. Anakinra treatment in macrophage activation syndrome: a single center experience and systemic review of literature. Clin Rheumatol. 2018;37(12):3329-35.

25. Gabay C, Lamacchia C, Palmer G. IL-1 pathways in inflammation and human diseases. Nat Rev Rheumatol. 2010;6(4):232-41.

26. Matsubara D, Kauffman HL, Wang Y, Calderon-Anyosa R, Nadaraj S, Elias MD, et al. Echocardiographic findings in pediatric multisystem inflammatory syndrome associated with COVID-19 in the United States. J Am Coll Cardiol. 2020;76(17):1947-61.

27. Mavrogeni SI, Kolovou G, Tsirimpis V, Kafetzis D, Tsolas G, Fotis L. The importance of heart and brain imaging in children and adolescents with multisystem inflammatory syndrome in children (MIS-C). Rheumatol Int. 2021;41(6):1037-44.

28. Sanil Y, Misra A, Safa R, Blake JM, Eddine AC, Balakrishnan P, et al. Echocardiographic indicators associated with adverse clinical course and cardiac sequelae in multisystem inflammatory syndrome in children with coronavirus disease 2019. J Am Soc Echocardiogr. 2021;34(8):862-76.

29. Salliot C, Dougados M, Gossec L. Risk of serious infections during rituximab, abatacept and anakinra treatments for rheumatoid arthritis: meta-analyses of randomised placebo-controlled trials. Ann Rheum Dis. 2009;68(1):25-32.

30. Kullenberg T, Löfqvist M, Leinonen M, Goldbach-Mansky R, Olivecrona H. Long-term safety profile of anakinra in patients with severe cryopyrinassociated periodic syndromes. Rheumatology. 2016;55(8):1499-506.

31. Klein A, Klotsche J, Hügle B, Minden K, Hospach A, Weller-Heinemann F, et al. Long-term surveillance of biologic therapies in systemic-onset juvenile idiopathic arthritis: data from the German BIKER registry. Rheumatology. 2020;59(9):2287-98.

32. Youssef J, Novosad SA, Winthrop KL. Infection risk and safety of corticosteroid use. Rheum Dis Clin N Am. 2016:42(1):157-76.

33. Kabeerdoss J, Pilania RK, Karkhele R, Kumar TS, Danda D, Singh S. Severe COVID-19, multisystem inflammatory syndrome in children, and Kawasaki disease: immunological mechanisms, clinical manifestations and management. Rheumatol Int. 2021;41(1):19-32.

34. Lee PY, Day-Lewis M, Henderson LA, Friedman KG, Lo J, Roberts JE, et al. Distinct clinical and immunological features of SARS-CoV-2-induced multisystem inflammatory syndrome in children. J Clin Invest. 2020;130(11): 5942-50.

35. Aydın F, Çelikel E, Ekici Tekin Z, Coșkun S, Sezer M, Karagöl C, et al. Comparison of baseline laboratory findings of macrophage activation syndrome complicating systemic juvenile idiopathic arthritis and multisystem inflammatory syndrome in children. Int J Rheum Dis. 2021; 24(4):542-7.

36. Payne AB, Gilani Z, Godfred-Cato S, Belay ED, Feldstein LR, Patel MM, et al. Incidence of multisystem inflammatory syndrome in children among US persons infected with SARS-CoV-2. JAMA Netw Open. 2021;4(6):e2116420.

\section{Publisher's Note}

Springer Nature remains neutral with regard to jurisdictional claims in published maps and institutional affiliations.

Ready to submit your research? Choose BMC and benefit from:

- fast, convenient online submission

- thorough peer review by experienced researchers in your field

- rapid publication on acceptance

- support for research data, including large and complex data types

- gold Open Access which fosters wider collaboration and increased citations

- maximum visibility for your research: over $100 \mathrm{M}$ website views per year

At BMC, research is always in progress.

Learn more biomedcentral.com/submissions 